$e_{p 0}^{t}$ and $g_{A}=1132 / 50.1=22.6$ for $e_{n_{1}}^{t}$ by comparing our data with those of Fairfield and Gokhale. Apart from experimental errors of about $\pm 10 \%$ in our data and $\pm 15 \%$ in $c_{n 0}^{t}$ and $\pm 30 \%$ in $c_{p 1}^{t}$ in Fairfield and Gokhale's data, the remaining difference of the two values of $g_{A}$ may in part be due to the electric field dependence of the emission and capture rates.

The field dependence of the measured emission rates is further illustrated by the data of the hole emission rate at the gold-donor level, $e_{p-1}^{t}$ in Table $\mathrm{I}$, which were taken at $10^{5} \mathrm{~V} / \mathrm{cm}$. A comparison of our result with the thermal equilibrium value of Fairfield and Gokhale given in the table shows that $g_{D}=3.88 \times 10^{7} / 7.67 \times 10^{5}=51$, which reflects further the electric-field enhancement of the thermal emission rate.

\footnotetext{
${ }^{1}$ W. Shockley and W. T. Read, Phys. Rev. 87, 835 (1952).

${ }^{2}$ C. T. Sah, Proc. IEEE 55, 654 (1967); 55, 673 (1967).
}

${ }^{3}$ J. M. Fairfield and B. V. Gokhale, Solid State Electron. 8, 685 (1965).

${ }^{4}$ G. Bemski, Proc. IRE 46, 990 (1958).

${ }^{5}$ M. Lax, Phys. Chem. Solids 8, 66 (1959); Phys. Rev. 119, 1502 (1960).

${ }^{6}$ J. Frenkel, Phys. Rev. 54, 647 (1938).

${ }^{7}$ C. T. Sah, L. Forbes, L. L. Rosier, and A. F. Tasch, Jr., Third International Photoconductivity Conference, August 1969 (unpublished); Thermal and Optical Emission and Capture Rates and Cross Sections of Electrons and Holes at Defect Centers in Semiconductors from Photo and Dark Junction Current and Capacitance Experiments (to be published).

${ }^{8}$ C. T. Sah and A. F. Tasch, Jr., Phys. Rev. Letters 19, 69 (1967); C. T. Sah, A. F. Tasch, Jr., and D. K. Schroder, ibid. 19, 71 (1967).

${ }^{9}$ A. F. Tasch and C. T. Sah (unpublished).

${ }^{10}$ C. B. Collins, R. O. Carlson, and C. J. Gallagher, Phys. Rev. 105, 1168 (1957).

\title{
STABLE, CHIRPED, ULTRASHORT PULSES IN LASERS USING THE OPTICAL KERR EFFECT*
}

\author{
J. C. Comly, A. Yariv, and E. M. Garmire \\ California Institute of Technology \\ Pasadena, California
}

(Received 9 June 1969; in final form 1 August 1969)

\begin{abstract}
A self-consistent analysis of pulse propagation inside a laser cavity containing, in addition to an amplifying medium, some material displaying the optical Kerr effect, yields ultrashort pulses. These pulses become stable in the limit of high line-center excess gain with the stabilization provided by an interplay between strong chirping in the Kerr medium and bandlimiting in the laser medium.
\end{abstract}

This paper presents an analysis of pulse propagation in a laser cavity containing some material displaying the optical Kerr effect, in addition to an amplifying medium. The question of mode locking a laser by means of the Kerr effect has been considered previously using a coupled mode approach ${ }^{1}$; in this paper we consider the same problem using the circulating pulse techniques developed by Cutler ${ }^{2}$ and applied recently to laser systems. ${ }^{3,4}$ We find that this approach enables us to predict, in addition to the existence of ultrashort pulses, their amplitude, pulse width, and chirp. The analysis also reveals the presence of strong pulse stability occurring in the limit of high line-center excess gain in the laser medium and strong chirping in the Kerr cell.

We consider a Gaussian pulse with chirp circulating in the laser cavity. Simplified models for the laser and Kerr media are adopted, allowing the pulse to remain Gaussian, so that closed-form ana-

*Work supported jointly by the U.S. Army Research Office-Durham, and by the U.S. Air Force of Scientific Research. lytical solutions can be obtained. Also, all constant phase terms and group delays are ignored, since we are not calculating the loop transit time. The physical interpretation of the results suggest, however, that qualitatively they do not depend on the simple model chosen.

The laser, shown in Fig. 1, is presumed to have a length $L_{0}$ of amplifying medium near one end, and a cell of length $L_{1}$ containing the liquid (or solid) Kerr medium near the other end. An optical pulse in the form of

$$
E_{1}(t)=A_{1} \exp \left[-\alpha_{1} t^{2}+i\left(\omega_{0} t+\beta_{1} t^{2}\right)\right]
$$

is assumed to be traveling from the Kerr cell toward the laser medium. If we neglect the overlap of a pulse with itself during reflection, we may represent one round trip as a traversal of a laser medium of length $2 L_{0}$ followed by a passage through a Kerr medium of length $2 L_{1}$.

The laser medium is characterized by a Gaussian transfer function. ${ }^{5}$

$$
g(\omega)=\exp \left\{2 a L_{0}\left[1-T_{2}^{2}\left(\omega-\omega_{0}\right)^{2}\right]\right\} .
$$




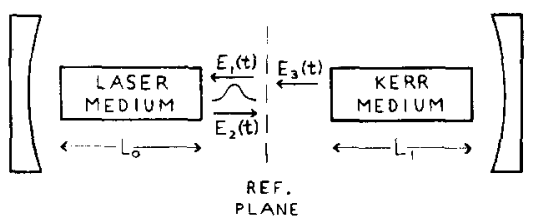

Fig. 1. Schematic of optical resonator showing gain (laser) medium and medium displaying optical Kerr effect.

$$
E_{2}(\omega)=E_{1}(\omega) G^{1 / 2} \exp \left[-\left(\omega-\omega_{0}\right)^{2} / 4 \alpha_{L}\right],
$$

where $\alpha_{L}=\left(8 a L_{0} T_{2}^{2}\right)^{-1}$ and $G=R \exp \left(4 a L_{0}\right)$ is the net round trip line-center power gain, with $R<1$ being the power loss factor per round trip. Using Eqs. (1) and (3) yields an expression for the pulse $E_{2}(t)$ at the output of the amplifying medium

$$
E_{2}(t)=A_{2} \exp \left[-\alpha_{2} t^{2}+i\left(\omega_{0} t+\beta_{2} t^{2}\right)\right],
$$

where

$$
\begin{gathered}
A_{2}=\frac{A_{1} G^{1 / 2}}{\left[\left(1+\alpha_{1} / \alpha_{L}\right)^{2}+\left(\beta_{1} / \alpha_{L}\right)^{2}\right]^{1 / 4}}, \\
\alpha_{2}-i \beta_{2}=\frac{\alpha_{1}\left(1+\alpha_{1} / \alpha_{L}\right)+\beta_{1}\left(\beta_{1} / \alpha_{L}\right)-i \beta_{1}}{\left(1+\alpha_{1} / \alpha_{L}\right)^{2}+\left(\beta_{1} / \alpha_{L}\right)^{2}} .
\end{gathered}
$$

The passage of the pulse through the Kerr liquid will generally result in distortions in both the envelope and phase functions. ${ }^{6}$ For conditions which are easily satisfied the envelope distortion will be small and the phase distortion can be approximated by an added chirp which is proportional to the pulse intensity and inversely proportional to the square of the pulse length. ${ }^{7}$ Within these approximations, the pulse after passing (twice) through the Kerr cell is given by

$$
\begin{aligned}
E_{3}(t) & =E_{2}(t) \exp \left(i C_{0} A_{2}^{2} \alpha_{2} t^{2}\right) \\
& =A_{3} \exp \left[-\alpha_{3} t^{2}+i\left(\omega_{0} t+\beta_{3} t^{2}\right)\right],
\end{aligned}
$$

where $C_{0}$ is a constant depending on the Kerr medium and its length. It follows directly that

$$
A_{3}=A_{2}, \quad \alpha_{3}=\alpha_{2}, \quad \beta_{3}=\beta_{2}+C_{0} A_{2}^{2} \alpha_{2} .
$$

The condition for "steady state" is that the pulse reproduce itself after one round trip. This requires that

$$
A_{3}=A_{1}, \quad \alpha_{3}=\alpha_{1}, \quad \beta_{3}=\beta_{1}
$$

which, using (5), yields equations whose solutions determine uniquely the intensity, duration, and chirp of the self-reproducing pulse. These are given by

$$
\begin{aligned}
& A_{0}^{2}=\frac{1}{C_{0}}\left(\frac{G^{2}-1}{G}\right) \simeq \frac{G}{C_{0}}, \\
& \alpha_{0}=\alpha_{L}\left(\frac{G^{2}-1}{G^{2}+1}\right) \simeq \alpha_{L}, \\
& \beta_{0}=\alpha_{L} G\left(\frac{G^{2}-1}{G^{2}+1}\right) \simeq \alpha_{L} G .
\end{aligned}
$$

The approximate relations hold in the case of high excess line-center gain, $G \gg 1$.

In the limit of small excess gain, $G \simeq 1$, the results are similar to those of Ref. 4, for active FM mode locking. The present paper is primarily concerned, however, with the effects of high gain $G \gg 1$. Here the pulse length tends toward the value

$$
T_{p}=\left(2 \alpha_{0}\right)^{1 / 2} \simeq\left(2 \alpha_{L}\right)^{-1 / 2}=\left(4 a L_{0}\right)^{1 / 2} T_{2}
$$

and is thus approximately equal to the reciprocal of the laser gain linewidth. The chirp at the output of the Kerr cell causes the frequency to sweep over a bandwidth

$$
(\Delta \omega)_{\max } \simeq 2 \beta_{0} T_{p} \simeq G / T_{p}
$$

which is $G$ times the gain linewidth.

We thus find that the presence of a Kerr medium inside the cavity in combination with a high profiled gain gives rise to ultrashort pulses with a duration equal to the inverse gain linewidth as characteristic of mode-locked pulses. Furthermore, a strong frequency chirp which is proportional to the power gain is produced, leading to the possibility of compression ${ }^{7}$ to a limiting duration equal to their inverse bandwidth given by $\left(T_{p}\right)_{\text {compressed }}=\left(T_{p}\right) / G$, i.e., a factor $G$ down from the inverse gain linewidth. As an example, using a value of $G=10$ and taking $T_{p}$ for $\mathrm{Nd}^{3+}$ : glass as $10^{-13} \mathrm{sec}$, we obtain a compressed pulse width of $\sim 10^{-14}$ sec.

The pulses as given in Eq. (8) in the high gain limit are very stable against pulse-to-pulse variations as shown by the following argument. For small changes in the pulse parameters, $A_{1}, \alpha_{1}$, and $\beta_{1}$ from the steady-state values $A_{0}, \alpha_{0}$, and $\beta_{0}$, the changes in the "next" pulse are given by

$$
\begin{aligned}
& \frac{\left(A_{3}^{2}-A_{0}^{2}\right)}{A_{0}^{2}} \simeq \frac{\left(\beta_{3}-\beta_{0}\right)}{\beta_{0}} \simeq \frac{\left(A_{1}^{2}-A_{0}^{2}\right)}{A_{0}^{2}}-\frac{\left(\beta_{1}-\beta_{0}\right)}{\beta_{0}}, \\
& \frac{\left(\alpha_{3}-\alpha_{0}\right)}{\alpha_{0}} \simeq 0 .
\end{aligned}
$$

These formulas result from partial derivative expansions and are correct to order $G^{-2}$; thus, two successive applications of these formulas indicate that any perturbation in the pulse from the steadystate values will be reduced by this factor of $G^{-2}$ within two round trips. The stability in $\alpha$ and $\omega_{0}$ was demonstrated by Cutler ${ }^{2}$ for a highly nonlinear cavity element, but the above stability in $A$ and $\beta$ exists only in the limit of high excess line-center gain using a Kerr medium.

Physically, the Kerr medium feeds energy into frequencies outside the gain linewidth and, thus, acts as an effective loss mechanism reducing the pulse round-trip gain to unity. The strong stability is present as long as the Kerr cell chirps the pulse over frequencies much broader than the gain linewidth and by an amount proportional to the pulse intensity (at least, for those frequencies near $\omega_{0}$ ); thus, reasonable distortions and relaxation-time ef- 
fects produced in the Kerr medium will not affect the stability argument. The limited bandwidth amplification is achieved with a laser medium coupled with a sufficiently large constant loss, $R \ll 1$. Under such conditions the output from the laser medium will be determined by the gain profile and be essentially independent of the input pulse shape.

If the gain profile is provided by a gain medium plus a limited bandwidth device having a linewidth narrower than the laser gain line, then the condition $R \leqslant 1$ can be relaxed, which would decrease the gain needed in the laser medium. Finally, it should be noted that any strongly nonlinear element in a laser cavity, plus a large excess laser gain should lead to pulses of the type discussed above; the strong stability occurs, however, only when the nonlinearity is proportional to the pulse intensity, as is the case with the Kerr effect.

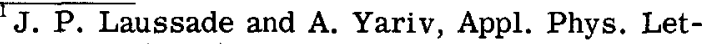
ters 13,65 (1968).
${ }^{2}$ C. C. Cutler, Proc. IRE 43, 140 (1955).

${ }^{3}$ A. J. DeMaria, W. H. Glenn, M. J. Brienza, and M. E. Mack, Proc. IEEE 57, 2 (1969).

${ }^{4}$ A. E. Siegman and D. J. Kuizenga, Appl. Phys. Letters 14, 181 (1969).

${ }^{5}$ Equation (2) shows an absorption or loss occurring when $T_{2}^{2}\left(\omega-\omega_{0}\right)^{2} \geq 1$. In the physical picture this is achieved by having a reflective loss greater than gain at these frequencies. Differences in the tails of $g(\omega)$ may give slightly different steadystate values than in (8), but will not affect the basic conclusions. In Eq. (2), a phase term $\exp \left[i T_{2}\left(\omega-\omega_{0}\right)\right]$ has been ignored (see Ref. 4), since it simply leads to a group time delay.

${ }^{6}$ F. DeMartini, C. H. Townes, T. K. Gustafson, and P. L. Kelley, Phys. Rev. 164, 312 (1967); T. K. Gustafson, J. P. Taran, H. A. Haus, J. P. Lifsitz, and P. L. Kelley, ibid. 177, 306 (1969).

${ }^{7}$ R. A. Fisher, P. L. Kelley, and T. K. Gustafson, Appl. Phys. Letters 14, 140 (1969).

\title{
ELECTRICAL CONDUCTION IN SINGLE-CRYSTAL ALUMINUM THIN FILMS*
}

\author{
T. S. Jayadevaiah \\ Department of Electrical Engineering \\ Institute for Surface Studies \\ University of Wisconsin-Milwaukee \\ Milwaukee, Wisconsin 53201 \\ Robert E. Kirby \\ Department of Physics \\ Institute for Surface Studies \\ University of Wisconsin-Milwaukee \\ Milwaukee, Wisconsin 53201 \\ (Received 29 May 1969; in final form 30 June 1969)
}

\begin{abstract}
Electrical conduction in thin (400-1500 $\AA$ ) single-crystal aluminum films vacuum evaporated on $\mathrm{NaCl}$ substrates is explained by a simple model and verified by experimental results. The resistivity of the inter-island boundaries is accounted for by considering the film structure as a metal-insulator matrix and calculating its equivalent resistivity. An unambiguous comparison can then be made with the FuchsSondheimer theory of size-affected conduction in thin metal films only after subtracting off the resistivity contribution due to interisland boundaries.
\end{abstract}

Recently there has been considerable technological interest in the properties of thin aluminum films as applied to thin film circuits. Resistivity and Hall coefficient data ${ }^{1}$ presently available are for films with a positive temperature coefficient of resistance (TCR) and thicknesses of several microns. These data are only in fair agreement with the Fuchs $-{ }^{2}$ Sondheimer ${ }^{3}$ (hereafter abbreviated as FS) theory of size-affected conduction processes in thin metal films. This letter reports a study on thinner (400-2000 $\AA$ ) films with positive TCR and approaching metallic conduction but having a resistivity greater than that of the bulk.

It appears logical, at first sight, that conduction size effects can be easily measured in thin evaporated films $(200-2000 \AA)$ since the surfaceto-volume ratio is sufficiently large. Investigations ${ }^{4}$ made in this direction have yielded unsatisfactory results compared to FS theory, in particular, large electron mean free paths. ${ }^{5}$ The assumption that the structure of films of varying thicknesses prepared in the same evaporation does not change is not usually valid in view of observations described below. This letter reports resistivity measurements of single-crystal Al films (400-2000 $)$ evaporated on $\mathrm{NaCl}$ substrates for various thicknesses and a simple calculation to show the size effects due to structure on the conductivity of these films.

A simple model first described by Volger ${ }^{6}$ and later used by Bube ${ }^{7}$ to explain Hall effects in in- 